\title{
Evaluating design pattern based solutions with their equivalent simpler solutions to promote maintainability in software
}

\begin{abstract}
This research mainly concentrates on investigating different design solutions which can be applied during the early design phase of software development, where these design solutions would likely reduce the latter maintenance costs. It requires measuring the maintenance level of a design solution during the design phase so as to minimize the cost, resources, and effort required. The solutions used in this research are design pattern based solutions and their alternative simpler solutions (i.e., solutions without using design patterns) so as to quantify which of them is more suitable towards improving the latter maintainability. A positivist research approach is used to conduct a quantitative experiment on the State and Proxy design patterns. This approach requires software engineers to measure the maintenance level of available solutions beforehand by using specific metrics made for this very purpose. As a result, the quantified values would assist us to select the most suitable design inclined towards reducing the maintenance costs and contribute in improving the overall software quality. The results showed that the design pattern based solutions have positive maintainability effects. The measurements and evaluation processes were formulated into a guideline, and an experimental-based evaluation was used for its testing.
\end{abstract}

Keyword: Design patterns; Maintainability metrics; Software maintainability; Software quality 